\title{
Fanconi Anaemia - A Rare Case Report
}

\author{
Aziz MA, Chowdhury MU, Khan R, Shah MS, Islam S \\ Department of Haematology, Bangabandhu Sheikh Mujib Medical University, Dhaka, Bangladesh \\ e-mail:azizfcps@yahoo.com
}

\begin{abstract}
Fanconi anaemia is a rare and most common form of inherited aplastic anaemia. It is mostly autosmal (except one $\mathrm{x}$ link) recessive disorder characterized by diverse congenital malformations, progressive pancytopenia, and predisposition to both haematological malignancy and solid tumours. Congenital malformation varies from patient to patient and may affect the skeletal system as well as organ systems. Highly variable phenotypic presentation with clinical menifestations makes difficult for diagnosis in some cases. Chromosomal breakage study induce by Mitomycin-C (MMC)/Diepoxybutane(DEB) provide a unique cellular marker for the diagnosis. The incidence of FA is approximately 1 to 5 per million. In Bangladesh, so far no study or even any case was reported. In this case report, a two years nine months old male child presented with generalized weakness, recurrent episodes of fever and physical deformities. It was found him short stature, microcephaly, trianguler face,generalized hyperpigmentation with café au lait spots,absent both thumbs with flexor deformity of both wrists. Peripheral smear found bicytopenia, bone marrow aspiration and biopsy showed hypoplastic marrow mildly elevated LDH, X-ray and USG showed bone and organ agenesis and chromosomal breakage study is also positive.
\end{abstract}

Key Words : Fanconi anaemia, Café au lait spot, chromosomal breakage study

\section{Introduction}

FA was first described in 1927 by the Swiss Paediatrician Guido Fanconi. It is a rare genetically inherited autosomal recessive disorder characterized by congenital malformations, progressive pancytopenia, cellular hypersensitivity to DNA-cross-linking agents, predisposition to acute myelogenous leukemia (AML) and other malignancies. ${ }^{1}$ The developmental and physical abnormalities may include hyperpigmentation, short stature, malformations of the thumb and forearms, skeletal anomalies, small head or eyes, renal problems, hearing defect, heart disease, gastrointestinal difficulties and hypogonadism. The incidence of FA is approximately 1 to 5 per million. ${ }^{2}$ This condition is more common among the people of Ashkenazi Jews, with carrier frequency of 1 in $89^{3}$, and black South Africans where the carrier frequency is 1 in $83 .{ }^{4}$

\section{Case Report}

A two year nine months old male child presented with generalized weakness, recurrent episodes of fever and physical abnormalities. He was born of seven months of intrauterine life due to oligohydromnios with foetal distress by LUCS. During birth there was absent thumbs of both hands with flexor deformity of both wrists. After birth till now all the milestones of development was delayed. Antenatally, mother had no specific complaints and was uneventful. There was a history of suffering with ALL and death of his only sibling. Physical examination revealed patient had short stature as height for age (H/A) was below 10th percentile. He also had triangular face, microcephaly, generalised hyperpigmentation with café au lait spots, flat thenar eminence, absent both thumbs with flexor deformity of both wrists (figure 1). 

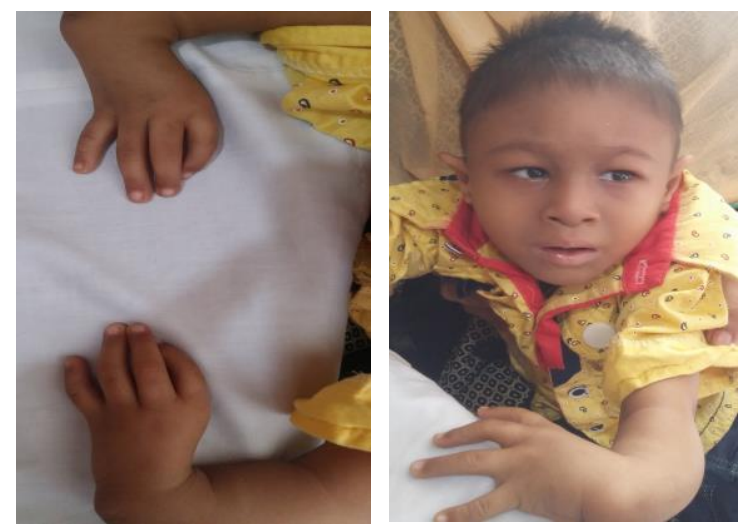

Figure 1: Absent thumb and Flexor deformity of both hand
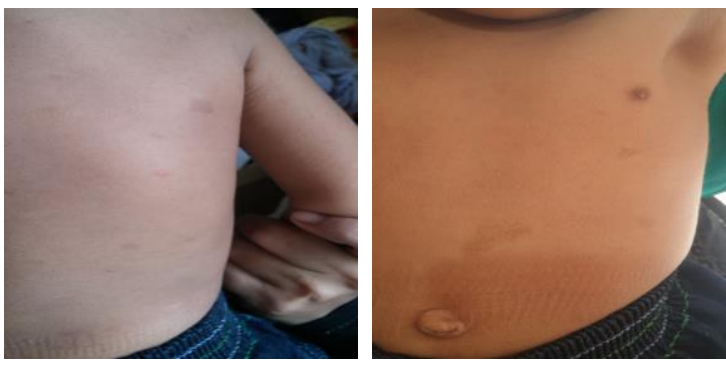

Figure 2: Hyperpigmented (café au lait) skin rash

Patient also had developmental delay and learning disability. Investigations revealed hemoglobin $7 \mathrm{gm} / \mathrm{dl}$, Hct $21.2 \%$, RBC Count 2.16 million/cumm, Total WBC count 6600/cumm, Platelet count <10x103/cumm. Liver and renal function tests were within normal limits LDH 797 U/L. Peripheral smear showed bicytopenia. Bone marrow aspiration and marrow biopsy showed hypocellularity with reduced erythroid, myeloid and megakaryocyte series cells suggestive of aplastic anemia (figure 3).

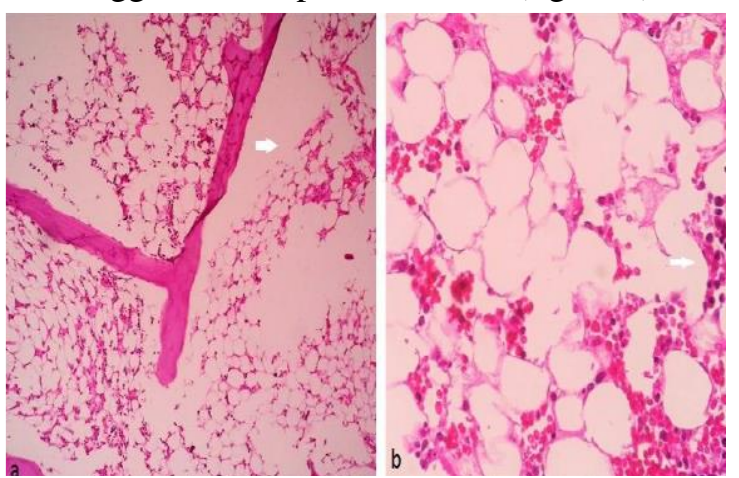

Figure 3 (a,b ): Hypoplastic marrow ( Trephine imprint a- $10 x$ and b- 40x)

USG of abdomen found absent left kidney,absent right testis and undescended left testis, X-ray both forearm found longitudinal pre-axial defects of both upper limbs with hypophalangism and also radial club hand deformity(figure 4).

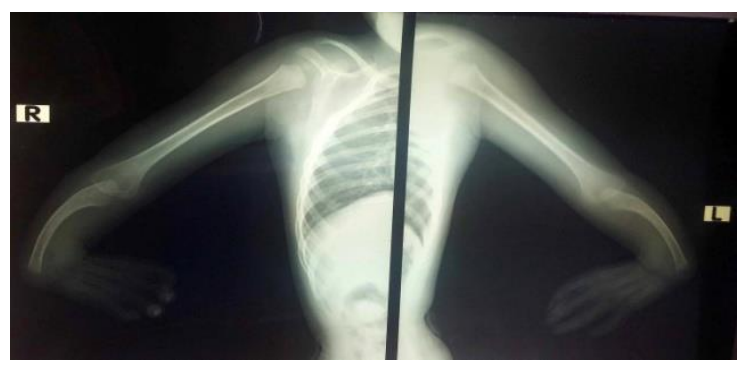

Figure 4: X-ray of hypophalagism with radial club hand deformity

Karyotyping found normal male karyotyping (46XY ). Chromosomal breakage study induce by Mitomycin-C found abnormal for chromosomal breakage syndeome with significant structural aberrations in most of the metaphases studied (in figure 5).

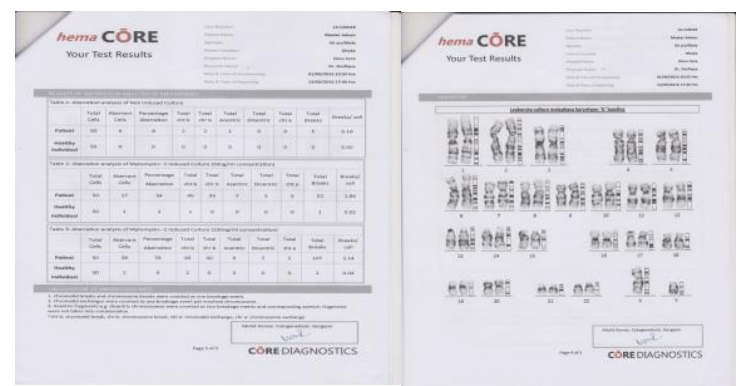

Figure 5: Chromosomal breakage study induce by Mitomycin-C found abnormal for chromosomal breakage syndeome with significant structural aberrations in most of the metaphases studied.

\section{Discussion}

Fanconi anemia (FA) is a genetically heterogeneous rare autosomal recessive disorder characterized by congenital malformations, hematological problems and predisposition to malignancies. This case was diagnosed based on physical abnormalities, blood investigations and bone marrow examination. Confirmatory tests are Chromosomal breakage studies where detection of chromosomal aberrations (breaks, rearrangements, radials, exchanges) in cells was done after culture with a DNA interstrand crosslinking agent such as DEB or MMC..$^{5}$

The differential diagnosis of FA based on clinical and laboratory evaluation includes Dyskeratosis 
congenita, Shwachman-Diamond Myelocerebellar disorder, Congenital amegakaryocytic thrombocytopenia, DNA ligase IV deficiency, Dubowitz syndrome, Nijmegen breakage syndrome, Reticular dysgenesis, Bloom syndrome, Seckel syndrome, VACTERL association and WT syndrome. ${ }^{6}$

The complications of fanconi anemia are bone marrow failure, acute myeloid leukemia, myelodysplastic syndromes and solid tumors of the head and neck, skin, gastrointestinal tract and genital tract. FA patients require transfusion red blood cells at frequent intervals. Other therapies include androgens to raise the hemoglobin and platelet count, G-CSF and GM-CSF for neutropenia Definitive treatment includes bone marrow transplant for marrow failure in FA patients. ${ }^{5}$ Preventive measures can be taken by prenatal testing and family planning. Prenatal testing includes fetal ultrasonography evaluation, molecular genetic testing by amniocentesis or chorionic villous sampling and chromosomal breakage studies with DEB/MMC. Family planning includes genetic counseling to young adults who are affected, carriers or at risk of being carriers. $^{7}$

\section{Conclusion}

It is concluded that genetic study should be done if possible in all the cases of suspected FA, siblings, parents and close blood relatives. The screening of the FANCA gene for mutations supports the clinical diagnosis of FA. Further, it will help to plan appropriate treatment and also to select suitable donor for hematopoietic stem cell transplantation and to plan for genetic counseling. Future studies would clearly advance the current understanding of FANCA regulation and function.

\section{References}

1. D'Andrea AD, Grompe M. The Fanconi anaemia/BRCA pathway. Nat Rev Cancer. 2003;3:23-34.

2. Joenje H, Patel KJ. The emerging genetic and molecular basis of Fanconi anaemia. Nat Rev Genet.2001;2:446-57.

3. Verlander PC, Kaporis A, Liu Q, Zhang Q, Seligsohn U, Auerbach AD. Carrier frequency of the IVS4 + 4 A®T mutation of the Fanconi anemiagene FAC in the Ashkenazi Jewish population. Blood.1995; 86:4034-38.

4. Timmers C, Taniguchi T, Hejna J, Reifsteck C, Lucas L, Bruun D, et al. Positional cloning of a novel Fanconi anemia gene, FANCD2. Mol Cell. 2001; 7:241-8.

5. Alter BP, Kupfer G. Fanconi anemia. Seattle: Gene reviews; 2002.

6. Lichtman MA, Kipps TJ, Seligsohn U et al. Williams Hematology. 8th ed, China: McGraw-Hill; 2010. Chapter 34, Aplastic anemia; p 586.

7. Department of Health and Research. XIIth Plan (2012-2017). New Delhi: Ministry of Health and Family Welfare, Government of India; 2012. Available from: URL: www.icmr.nic.in/.../plan/ ICMR\%20XIIth\%20Plan\%20(2012-2017).pdf 